\title{
Acupuncture Emergency Service in Brazilian Public Health System: Quantitative Analysis of Cases Attended in a Semester
}

José Udevanier Rebouças da Silva Júnior $\mathrm{MD}^{1 *}$, Lorena Anunziato Sant'Ana $\mathrm{MD}^{1}$, Mary Clea Ziu Lem Gun $\mathrm{MD}^{1}$, João Roberto Bissoto $\mathrm{MD}^{2}$, Ysao Yamamura MD, $\mathrm{PhD}^{3}$, Marcia Lika Yamamura MD, $\mathrm{MSc}^{4}$ and Silvana Maria Silva Fernandes $\mathrm{MD}, \mathrm{PhD}^{5}$

${ }^{1}$ Resident Physician, Acupuncture Medical Residency Program at Paulista School of Medicine, Federal University of São Paulo-EPM/UNIFESP, Brazil ${ }^{2}$ Acupuncturist Physician, Specialist by the Brazilian Medical Association (AMB), Preceptor of the Medical Residency Program in Acupuncture at the Paulista School of Medicine of the Federal University of São Paulo-EPM/UNIFESP, Brazil

${ }^{3}$ Acupuncturist Physician, Specialist in Acupuncture by the Brazilian Medical Association (AMB). Professor and Vice-Head of the Chinese Medicine Group - Acupuncture of the Department of Orthopedics, DOT at Paulista School of Medicine, Federal University of São Paulo-EPM/UNIFESP. PresidentDirector of the Center for Research and Study of Chinese Medicine - Acupuncture (Center AO), Brazil

${ }^{4}$ Acupuncturist Physician, Specialist in Acupuncture by the Brazilian Medical Association (AMB). Pediatrician. Master of Science of EPM/UNIFESP. Head of the Chinese Medicine Group - Acupuncture, Department of Orthopedics, DOT, Paulista School of Medicine, Federal University of São Paulo-EPM/ UNIFESP. Coordinator of the Specialization and Development Course in Chinese Medicine - Acupuncture at Center AO, Brazil

${ }^{5}$ Acupuncturist Physician, Specialist in Acupuncture by the Brazilian Medical Association (AMB). PhD in Sciences by the Department of Gynecology at UNIFESP. Coordinator of the Medical Residency Program in Acupuncture at Paulista School of Medicine, Federal University of São Paulo-EPM/ UNIFESP, Brazil

*Corresponding author: José Udevanier Rebouças da Silva Júnior, Federal University of São Paulo UNIFESP, Department of Orthopedics and Traumatology-Chinese Medicine-Acupuncture Group 715 Napoleão de Barros Street - 1st Floor - Vila Clementino, São Paulo - SP, Brazil; Tel: +55 (11) 5576-4848, +55 (85) 99983-3554; Branch line 3006; E-mail: udevanier@outlook.com

Received: October 08, 2021; Accepted: November 26, 2021; Published: November 29, 2021

\begin{abstract}
Background: Acupuncture is an effective technique for pain relief and is usually practiced in outpatient clinic setting. It can also be applied in emergency setting focusing on pain relief from non-life threatening diseases.

Objectives: This quantitative, retrospective and descriptive study aimed to demonstrate the dynamics of the Acupuncture Emergency Service at Hospital São Paulo (AES-HSP), linked to the Paulista School de Medicine of the Federal University of São Paulo (Escola Paulista de Medicina - EPM / UNIFESP), which provides free care for the population since 1998.
\end{abstract}

Methods: data were collected from the care records of the second half of 2019, assessing gender, age group, complaint, technique (s) used, percentage of improvement reported by the patient and Visual Analogue Scale before (VASb) and after treatment (VASa).

Results: we identified 7647 visits, of which $78.3 \%(n=5986)$ were female; the mean age was $60.8 \pm 14.3$ years-old; the most common complaints were low back pain $(26.4 \%)$, followed by shoulder pain $(17.5 \%)$ and knee pain $(14.8 \%)$; systemic acupuncture was used in a total of 7032 cases, only acupuncture microsystems were used in 615 cases, microsystems and systemic acupuncture were combined in 1815 cases; VASb average was $6.29 \pm 2.17$, while VASa average was $1.44 \pm 1.42$; in $21.4 \%$ of 6423 visits properly registered, patients reported $100 \%$ improvement and $72.2 \%$ reported more than $50 \%$ improvement.

Conclusion: our service provides effective pain relief, allowing to receive a great demand from patients with fast execution in an emergency setting, reducing the use of pain killers and its side effects.

Keywords: Acupuncture analgesia, Traditional Chinese Medicine, Public health, Pain Control

\section{Introduction}

Musculoskeletal pain (MSP) is classified as acute or chronic, and is the most prevalent symptom in the world population. Its prevalence has increased in recent years due to higher prevalence of risk factors related to lifestyle habits, such as smoking, anxiety, physical inactivity, sleep disorders. Additional influences include low educational level, precarious family income and social isolation [1,2]. In addition, MSP represents an important cause of morbidity, with a large impact on quality of life and in the economic sphere, for example, absence from work, sometimes requiring long periods of recovery [3]

European data related that $15-20 \%$ of primary health care appointments are due to musculoskeletal problems [4]. 
In Brazil, a meta-analysis performed in 2012 estimated the prevalence of chronic MSP ranging from 14.1-85.5\%. Considering only Brazilian studies were evaluated in the meta-analysis, the most affected sites were the dorsal spine and the lower limbs [5].

The impact of chronic pain on national economy also reaches a large proportion. For example, in 2007, Australia, a country with approximately 22.7 million inhabitants, had an estimated cost of $\$ 34.3$ billion for expenses related to chronic pain, with an average of $\$ 10,847$ per person with chronic pain [6].

The western medicine approach to MSP is mainly based on the use of common analgesics, opioids, anti-inflammatories and physical therapy. Allopathic drugs, however, are not exempt from adverse effects [7]. In addition, the presence of comorbidities, such as high blood pressure, diabetes, and chronic kidney disease, may restrict the use of such medications. Moreover, the inadequate follow-up of prescriptions and the practice of self-medication predispose to the overuse of anti-inflammatory drugs, which may cause serious complications, such as acute renal dysfunction, upper gastrointestinal bleeding due to peptic ulcer disease and occurrence of cardiovascular events [8-10]. The excessive use of opioids, in turn, might result in an increasing number of drug overdose, addiction and deaths [7].

Acupuncture has an energetic propaedeutic role, capable of detecting and treating an individual's imbalances before they evolve into organic diseases. In addition to the preventive aspect, it is an effective and safe therapeutic tool for many diseases [11].

The mechanism of action of acupuncture involves stimulation of peripheral nociceptors at specific points, which reach the nervous system through neuronal pathways. Neuromodulation occurs at three levels: local, spinal and supraspinatus, resulting in the release of different substances, such as neurotransmitters, that modulate motor, sensory, autonomic, neuroendocrine and emotional responses [12]. Is important to achieve the $\mathrm{Te} Q \mathrm{Q}$ needling sensation, characterized as a set of sensations, such as pain, burning, tingling, pressure, weight, anesthesia and/or shock, directly related to clinical efficacy [13].

In the west, the growing demand for acupuncture treatment is due to its effectiveness in pain complaints, especially in individuals with limitations to traditional pharmacological treatment [14].

In Brazil, the practice of acupuncture was introduced for the first time in SUS in 1999, through Ordinance No. 1230/GM [15], and was reinforced by its inclusion in the National Policy of Integrative and Complementary Practices (PNPIC), published in Ministerial Ordinance No. 971 of May 2006 [15].

In 1992, the Chinese Medicine-Acupuncture Group of the Department of Orthopedics and Traumatology at Paulista School of Medicine of Federal University of São Paulo (EPM/UNIFESP) was created by Ysao Yamamura M.D., PhD. This physician established this group to foment academic undergraduate and graduate activities, including clinical research, of the institution.

Initially, the therapeutic proposals were exclusively provided on an outpatient basis, resulting in great demand by the population, with an average number of 90 patients daily. Due to increasing demand, it was necessary to establish a more dynamic service. The AES-HSP, characterized by providing public assistance predominantly focused on analgesia under free demand access, was opened in 1998.

The AES-HSP team is composed of resident physicians, preceptors, graduate students and interns in the Chinese Medicine-Acupuncture Group. We have four patient care rooms in the outpatient clinic building of Hospital São Paulo (HSP), located in the Vila Clementino neighborhood in the city of São Paulo, state of São Paulo, Brazil. Clinic is held Monday to Friday from 8 am-3 pm, except on holidays. Patients are referred by basic health units or present directly; they are attended to based on arrival order.

In our service, a minimum number of acupuncture points with immediate effect of analgesia is used, with emphasis on the Yamamura System techniques of Acupuncture (SYA/EPM).

Microsystems, or somatotopies, are representations of the entire organism in smaller areas of the body. When the organism is sick, reactive points emerge in the microsystem in the areas corresponding to the compromised region. Through the manipulation of these reflex points, it is possible to act positively on the disease or symptomatology in question. In our service, we use internationally-renowned techniques, such as Yamamoto New Scalp Acupuncture (YNSA), Chinese Scalp Acupuncture and Chinese Auriculotherapy, as well as exclusive techniques developed by Dr. Yamamura [16-18] including the Yamamura Nasal Bone Acupuncture System (Figure 1), Yamamura Acupuncture System Hair Implantation (SYALIC) (Figure 2), Yamamura Long Bone Acupuncture System (SYAOL) (Figure 3), Yamamura Occipital Bone Acupuncture System (Figure 4), Yamamura System of Cranial Sutures and 5 Zang in parietal suture (Figure 5). Some of the main techniques are described below, and may be used isolated or associated with systemic acupuncture. Image of the systems of the Yamamura Acupuncture System were kindly provided by Dr. Yamamura.

\section{Methods}

We collected data from the attendance records at the AES-HSP, between July-December 2019, using a standardized form completed by the attending physician. The parameters evaluated included gender, age group, complaint, technique(s) used, percentage of improvement reported by the patient and Visual Analogue Scale before treatment (VASb) and after treatment (VASa).

We considered the total number of visits, not discriminating whether the same patient was seen on more than one occasion, and the main and associated complaints. Regarding treatment, we grouped the different approaches into isolated systemic therapy, non-systemic techniques or a combination of both.

A focused anamnesis and physical examination was performed for each patient and was directed to the patient's complaint in order to correctly select treatment points and techniques. Local asepsis was performed with cotton soaked in $70 \%$ alcohol, and sterile, disposable, $0.30 \mathrm{~mm}$ x $40 \mathrm{~mm}$ stainless steel acupuncture needles supplied by HSP were used. Needle insertion at specific points was performed until the $\mathrm{Te} Q \mathrm{Q}$ sensation was obtained, according to the depth characteristics. In auricular acupuncture, we used mustard seeds affixed to tape and manipulated with the aid of surgical tweezers. 


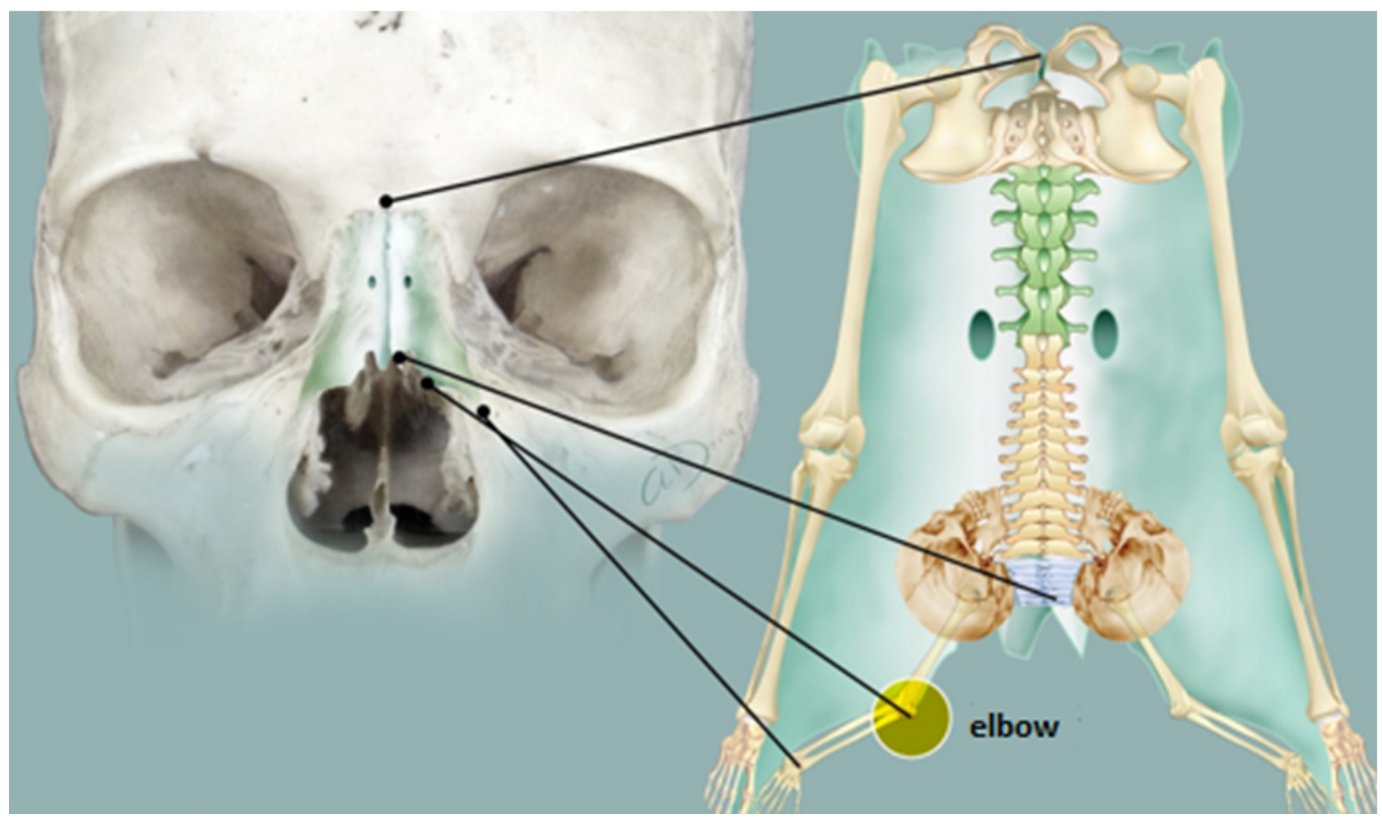

Figure 1: Yamamura acupuncture system of nasal bone.

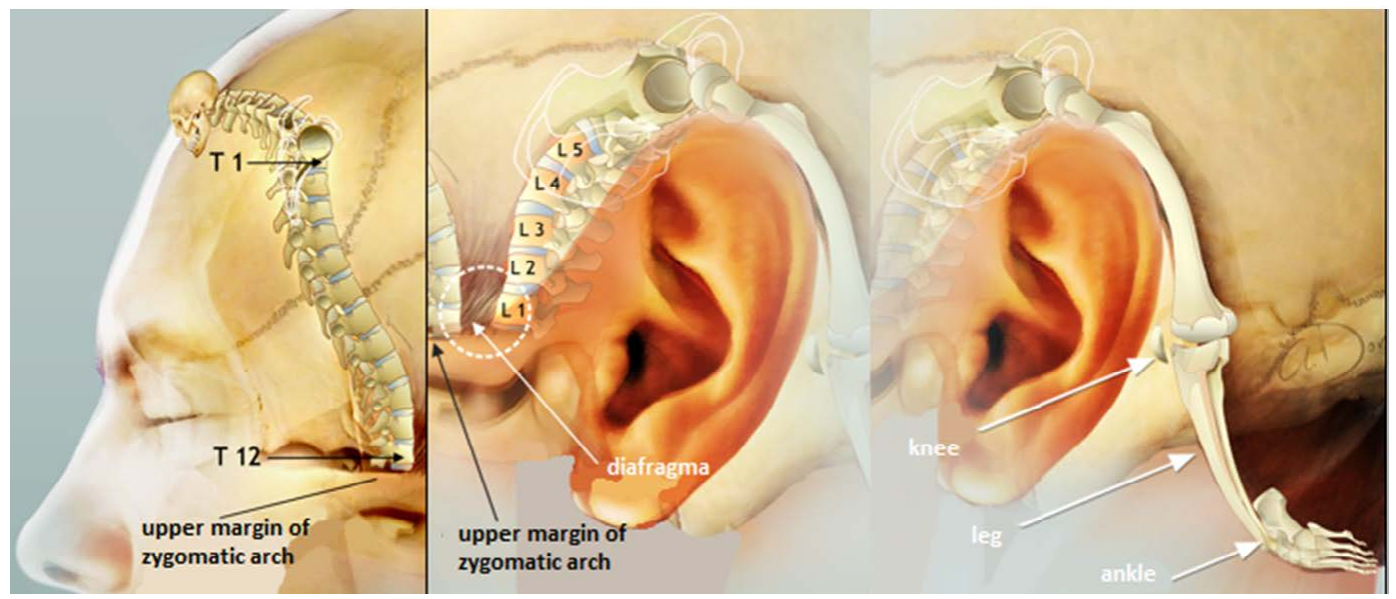

Figure 2: SYALIC - Yamamura acupuncture system of hair implantation line.

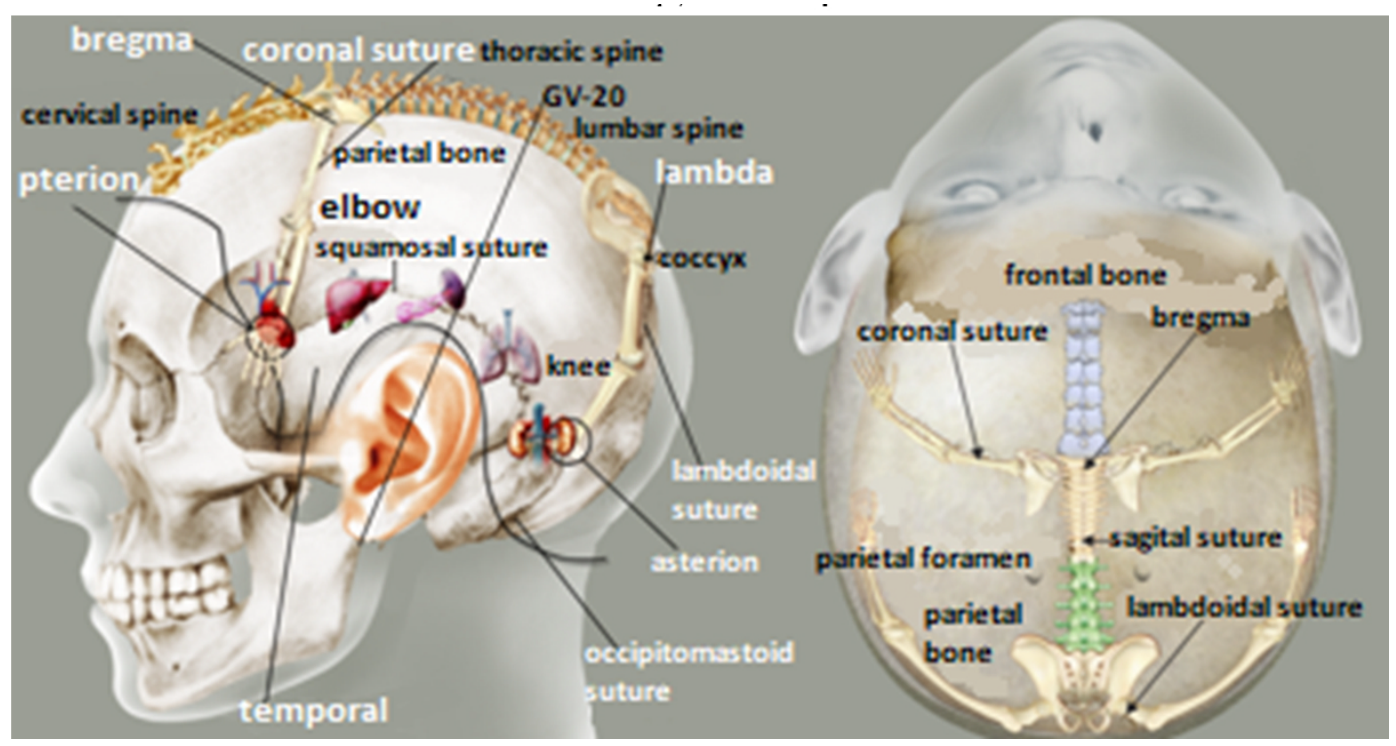

Figure 3: Yamamura acupuncture system of cranial sutures and 5 Zang on squamous suture. 


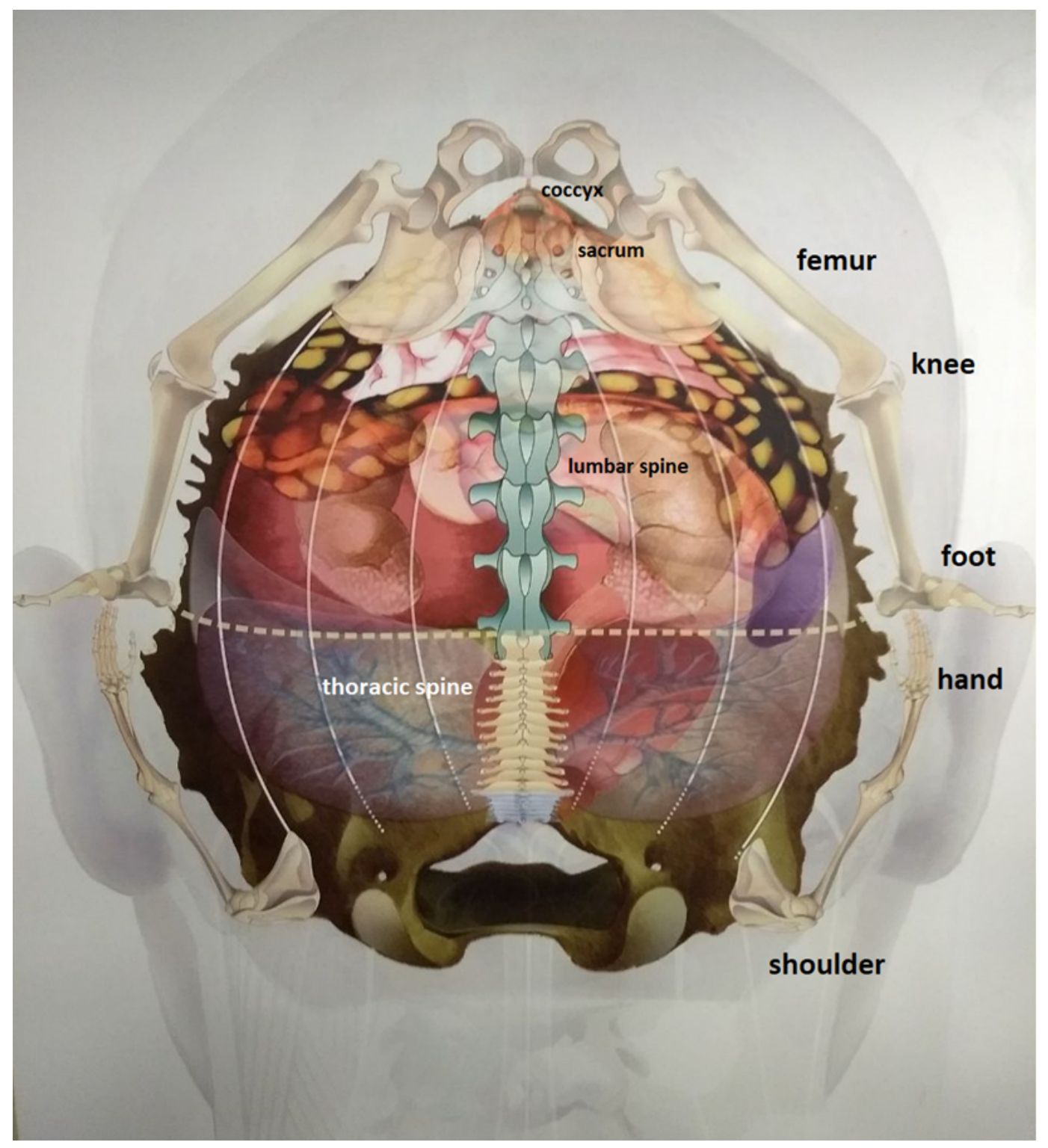

Figure 4: Yamamura acupuncture system of occipital bone.

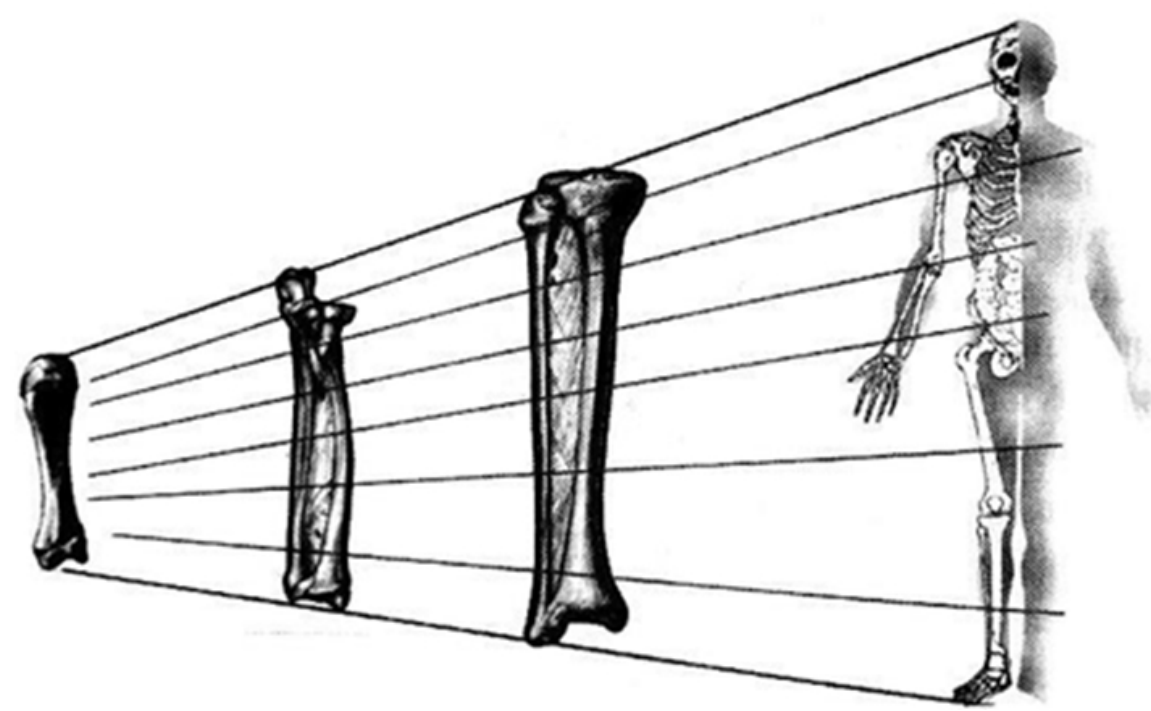

Figure 5: SYAOL - Yamamura acupuncture system of Long bone. 
Statistical analysis was performed descriptively, denoting average, median, minimum and maximum values, standard deviation, absolute and relative frequencies in percentage (\%), using Microsoft Excel ${ }^{\triangleright} 2019$ software by Microsoft. The graphs of columns and lines were elaborated using Microsoft PowerPoint ${ }^{\circledR} 2019$ software by Microsoft.

\section{Results}

We identified 7,647 visits, of which 78.3\% ( $\mathrm{n}=5986)$ were female and $21.7 \%(n=1661)$ were male. Regarding the age group, the mean age was (mean \pm standard deviation) $60.8 \pm 14.3$ years, with a median of 64 years; $85.7 \%$ of participants were between $41-80$ years (Graph 1), with a predominance in the range of 61-80 years, with a value of $53.4 \%$. The average number of visits corrected for working days in the semester (120) was 63.7 visits/day. The average number of patients per operating time (7 hours) was approximately 9.1 patients/hour, resulting in a duration of care of approximately 6.5 minutes/patient.

Regarding complaints, low back pain (26.4\%), followed by shoulder pain (17.5\%), knee pain (14.8\%), neck pain (11.7\%), upper back pain (5.9\%), lower limb pain (5.4\%), upper limb pain (4.9\%), foot pain (4.9\%), polyarthralgia (4.3\%), hip pain (2.7\%), polymyalgia $(2.3 \%)$, wrist pain $(1.3 \%)$, non-restorative sleep $(1.3 \%)$, hand pain $(0.9 \%)$, finger pain $(0.7 \%)$, facial palsy $(0.7 \%)$ and ankle pain $(0.5 \%)$. This data is depicted in Graph 2.

In the analysis of the VAS, the data referring to the index in VASb $(n=4080$ visits) corresponds to an average of 6.29 , with a median of 6 and standard deviation of 2.17. For the index in VASa $(n=3913)$, there was an average of 1.44 , median of 1 and standard deviation of

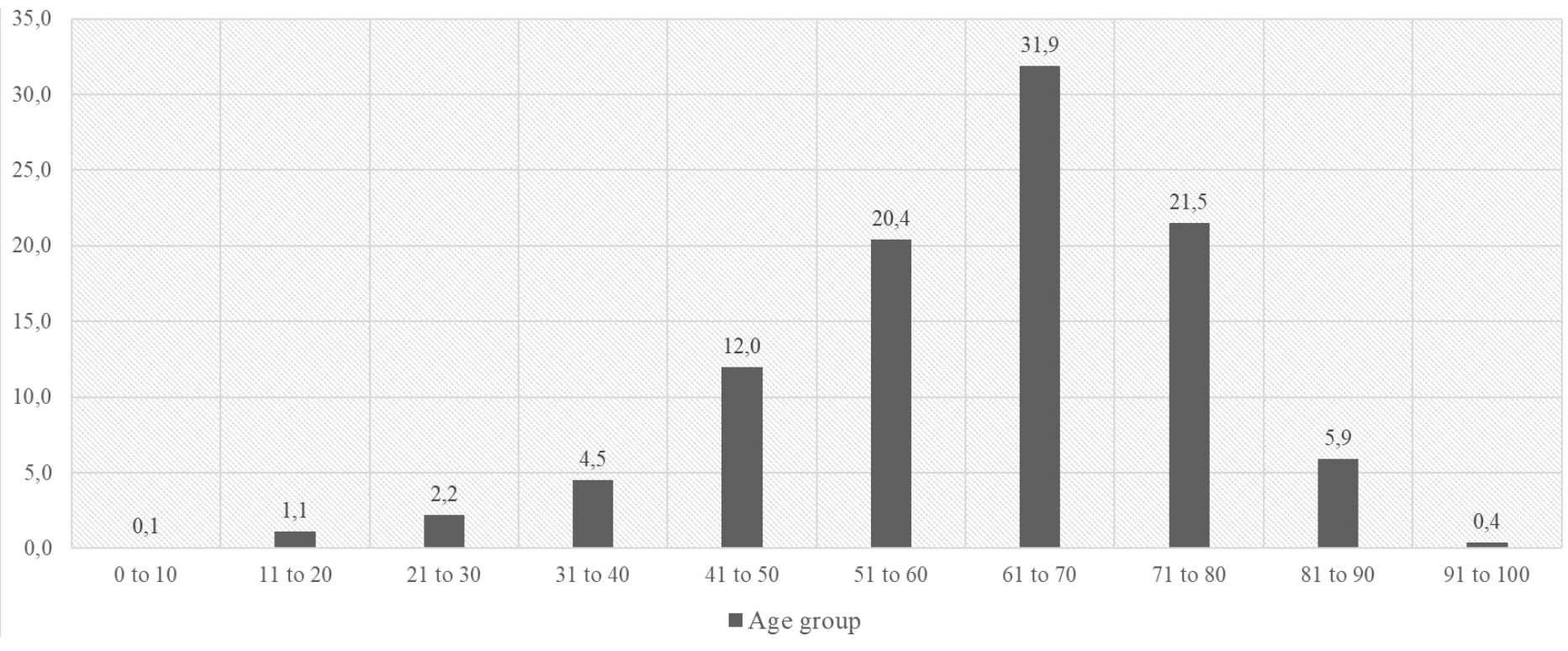

Graph 1: Distribution by age group (\%) $(\mathrm{n}=7647)$.

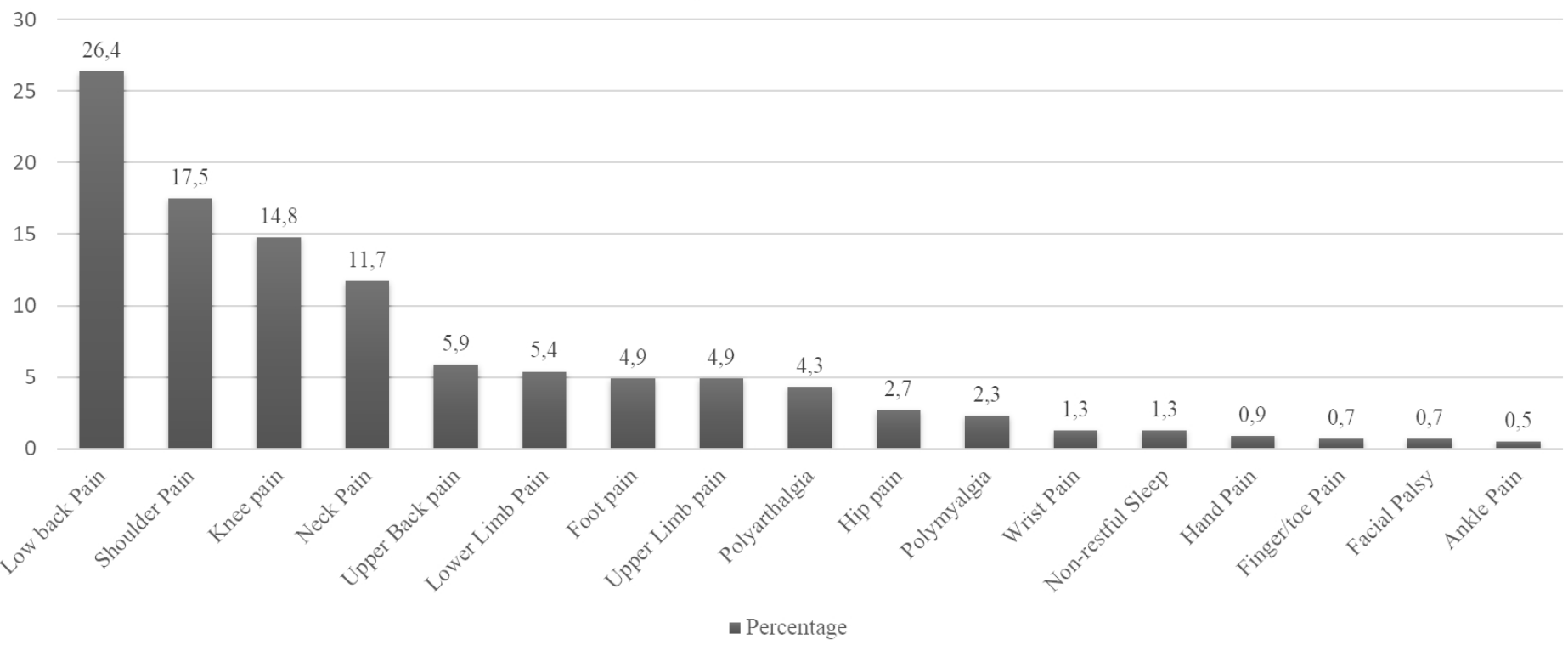

Graph 2: Percentage of most prevalent pain sites. 
1.42. There was a failure to register $46.6 \%(n=3567)$ of the VAS in relation to the total number of cases in the semester. The total visits $(n=6423)$ analyzed from the perspective of the degree of response to the treatment perceived by the patient were grouped into five categories: worsening $(0 \%)$, without improvement $(2 \%)$, less than $50 \%$ improvement $(4.3 \%)$, more than $50 \%$ improvement $(72.2 \%)$ and $100 \%$ improvement $(21.4 \%)$. This information is presented in Graphs 3 and 4.

Considering the total number of visits, non-systemic techniques were used 2,430 times. These techniques included: the Bregma craniometric point (22.2\%), Anatomical Trains (14.6\%), Auriculotherapy (13.2\%), Yamamoto New Scalp AcupunctureYNSA (12.1\%), Pterion craniometric point (9.1\%), Symmetry (7.8\%), Lambda Craniometric point (6.7\%), Asterion craniometric point (4.6\%), 5 Zang in parietal suture (2.8\%), Yamamura Acupuncture System Hair Implantation-SYALIC (1.7\%), Yamamura Long Bone Acupuncture System-SYAOL (0.8\%), Yamamura Occipital Bone Acupuncture System (0.5\%), Yamamura Nasal Bone Acupuncture System ( 0.4\%), Yamamura Acupuncture System of the Musculoskeletal System of Sutures (0.4\%), Vertebral Points $(0.3 \%)$, and Chinese Scalp Acupuncture (0.1\%) [15-17]. Graph 5 depicts this information.

Systemic acupuncture techniques were used in 7,032 cases, corresponding to $91.9 \%$ of total cases. The use of non-systemic techniques alone occurred in 615 cases, corresponding to $8 \%$ of the total. Microsystems and systemic acupuncture were combined in 1815 cases (23.7\%).

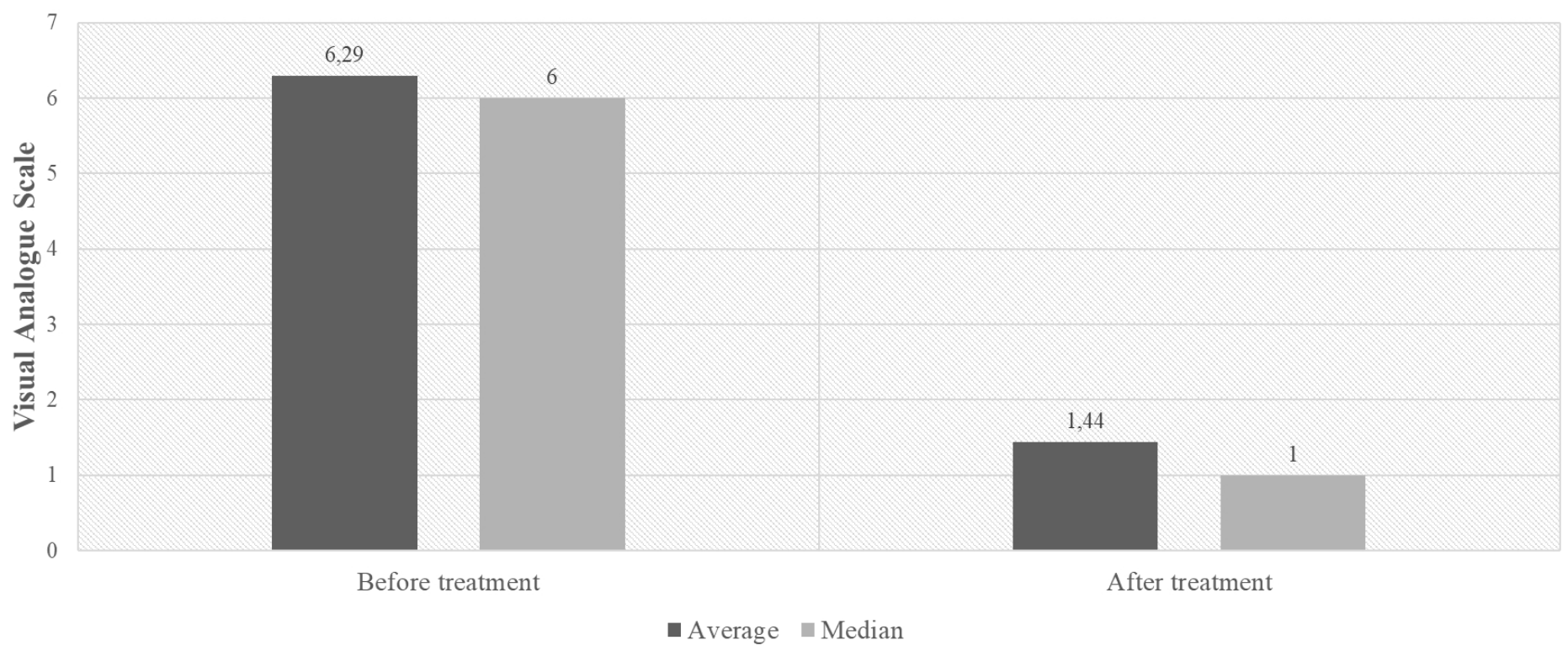

Graph 3: VAS before treatment (VASb) and after treatment (VASa).

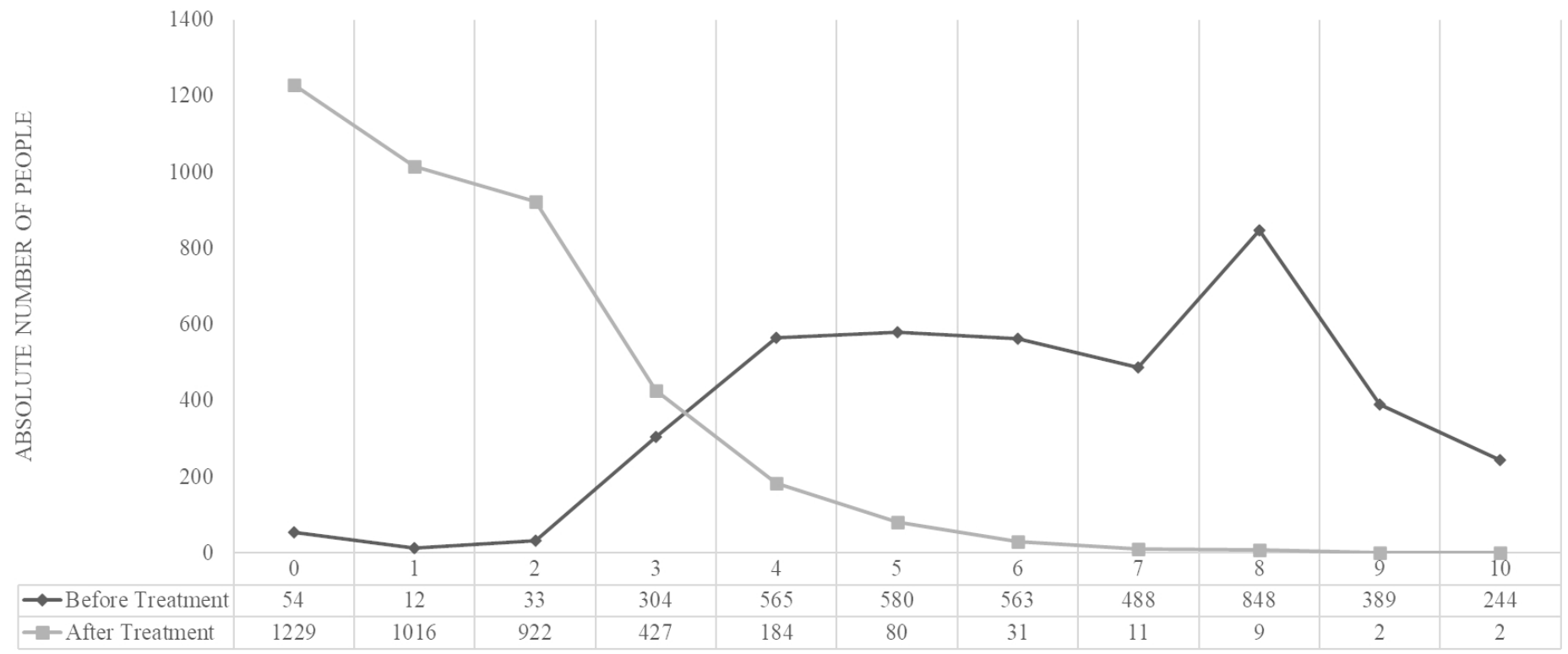

Graph 4: Continuous comparison of pain level before treatment (VASb) and after treatment (VASa). 


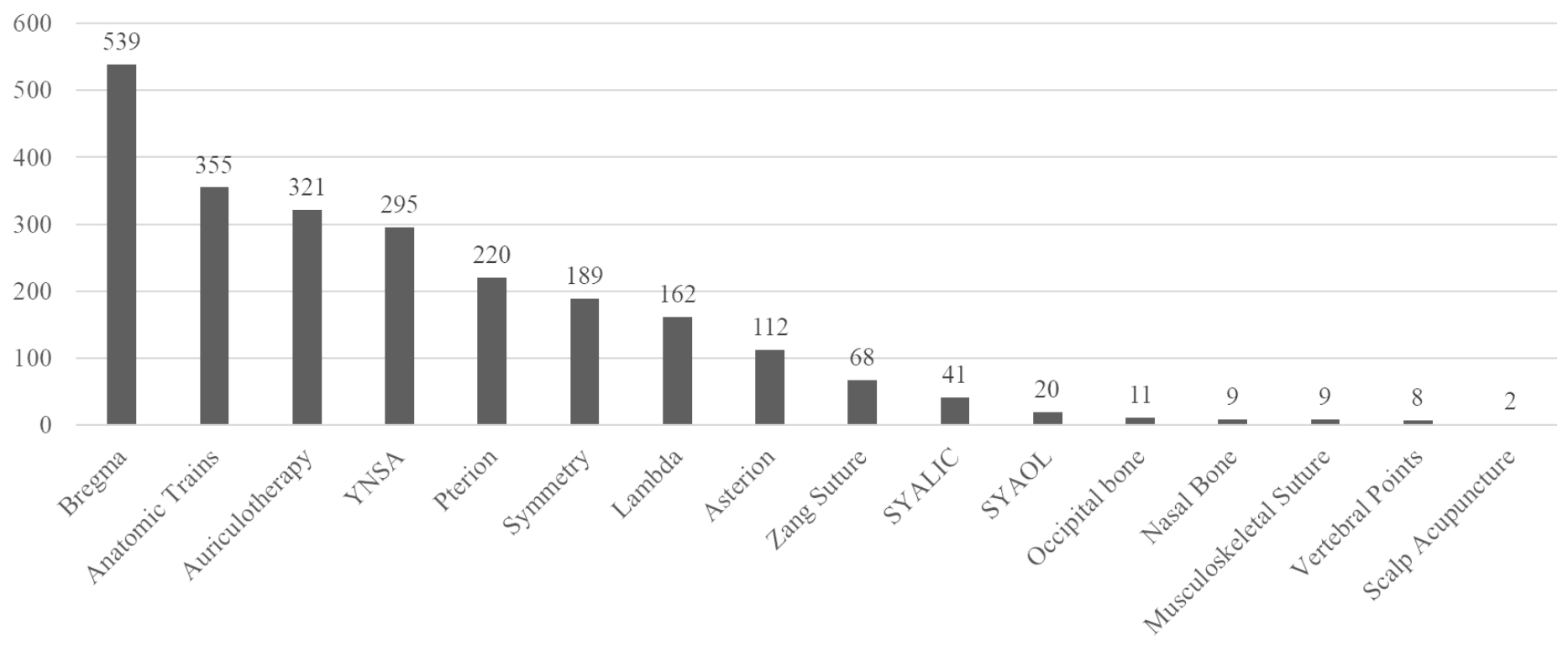

Graph 5: Distribution of non-systemic techniques most used ( $\mathrm{n}=2430)$.

\section{Discussion}

Our study identified a female prevalence rate three times higher than males. This information is in agreement with other studies, which state that the prevalence of women reporting chronic pain is generally higher than men, which can be influenced by the way men and women experience pain [19]. Another possible explanation is the social expression of each gender. Women are usually taught to express emotions and seek help, while men are generally inhibited from expressing themselves [20]. Thus, male patients are less likely to report chronic pain and seek medical assistance.

When we analyzed age group, we found that more than half of patients were between 41-80 years of age. According to the literature, older patients have a higher prevalence of chronic pain than younger patients. This may result from the increase in number of comorbidities presented in the elderly [21].

According to the Global Burden of Disease Study in 2016, low back pain and neck pain are the main causes related to disability worldwide [22]. Another study highlights low back pain as the main cause of disability globally [23]. The present study is in agreement with this worldwide incidence since the most frequent complaint was low back pain. Regarding shoulder pain, we found involvement in $17.5 \%$ of individuals. This data differs from the Brazilian meta-analysis by Miranda et al. (2012) that assessed the prevalence of musculoskeletal disorders in the elderly population in Brazil and found the spine as the most affected location and the lower limb the second-most affected [5]. Chronic knee pain presented as an important highlight in the visits, since it was the third most prevalent complaint.

Patients suffering from chronic pain often have more than one affected site, as demonstrated in a British demographic survey, in which only one-third of the participants with pain had localized symptoms [2]. Thus, in the prevalence chart of the most frequent complaints, the statistics of different sites of pain must be interpreted separately, only in relation to the total number of cases once the sum of painful sites exceeds the number of visits, because patients usually had more than one complaint.

The VAS was chosen to assess the degree of pain before (VASb) and after (VASa) the treatment with acupuncture because it is a validated instrument of easy applicability and reproducibility, low cost, and widely used in global literature, which allows comparison of the results $[24,25]$. Despite the failure to complete the VAS in almost half of patient visits, it was possible to perceive a clear reduction in the degree of pain, according to the mean and median between VASb and VASa registered in the graphs, implying an effective analgesia with acupuncture. Such efficacy was also reinforced by the degree of improvement reported by patients.

The failure to register VAS can be explained by the fact that it is an academic service and has a considerable turnover of people who required a new adaptation to the routine of functioning and data recording. Another possibility is the socioeconomic level of many patients who had difficulties understanding the VAS and unable to adequately grade their pain, occurring often enough to compel the attending physician to only ask about degree of pain improvement.

As it is an Emergency Service, highly effective techniques with few acupuncture points and manual stimulation are recommended, in the goal of obtaining a good response in a short period of time. In this way, microsystems are a very effective tool for simplicity in application and good resolution to pain, as well as in the selection of traditional systemic points of high effectiveness. The Chinese MedicineAcupuncture Group has developed treatment techniques validated by wide use in the AES-HSP that has proven to be highly effective, with some points being more used than traditional microsystems. Of the total number of consultations, the use of non-systemic techniques occurred 2,430 times, either alone or in combination with systemic points. The most used point for treatment was one of the craniometric points idealized by the Chinese Medicine-Acupuncture Group, Bregma, which was used in a total of 539 visits. 
Patients are aware of the service we offer, based on referral from general practitioners, family doctors and specialists, or through information obtained from acquaintances who have previously been assisted or the internet. As a result, they directly seek care, which assists a large population of patients awaiting care in outpatient clinics, where there is often a waiting list with months of delay. Due to the volume of patients, we had to consider the total number of visits, and new patients were not distinguished from return patients.

One of the difficulties with the present work was the lack of standardization of completing the attendance forms by the doctors of the service, resulting in some missing information, as occurred in the registration of the VAS. During the transfer of information in written form to Microsoft Excel ${ }^{\circ}$, the complaints were summarized in the key terms that motivated the patient visit in order to facilitate statistical analysis in the evaluation of the cases, which could represent a registration bias due to data simplification. However, this may be counteracted by the transcribing physician, who performs the role of organizing symptoms and signs in validated medical terms.

The services provided to these patients is important due the provision of immediate pain relief, reducing the demand for patients with chronic pain to utilize other emergency services. As a result, the physical and emotional impact of pain on patients' work and personal routine are minimized. The AES-HSP also reduces the time patients spend obtaining non-pharmacological pain therapy, for example, as in the Brazilian public unified health system, which has a waiting list for physiotherapy, which is another approach commonly used to manage MSP.

\section{Conclusion}

Acupuncture treatment to acute and chronic pain may reduce the use of self-medication, what decreases the risks of side effects of pain killers.

Our acupuncture emergency service provides effective care in pain relief in a fast and focused manner, resulting in significant demand from patients. Over its 22 years of existence, the services of the AESHSP is considered an alternative approach to provide analgesia to patients with chronic pain and serves as a model for the creation of new emergency care in acupuncture in public health systems.

\section{Authors' Contributions}

José Udevanier Rebouças da Silva Júnior M.D., Lorena Anunziato Sant'Ana M.D., and Mary Clea Ziu Lem Gun M.D. wrote the manuscript, constructed the graphs and translated image subtitles to English.

João Roberto Bissoto M.D., Ysao Yamamura M.D., PhD., Marcia Lika Yamamura M.D., MSc., and Silvana Maria Silva Fernandes M.D., $\mathrm{PhD}$. reviewed the manuscript, assisted in writing the manuscript and are supervisors of our Medical Residency Program.

Ysao Yamamura M.D., $\mathrm{PhD}$. is also the author of many techniques (microsystems) used in our service and owner of the pictures of the microsystems.

\section{References}

1. Bergman S, Herrström P, Högström K, Petersson I.F, Svensson B, Jacobsson L.T. (2001) Chronic musculoskeletal pain, prevalence rates, and sociodemographic associations in a Swedish population study. The Journal of Rheumatology 28: 136977. [crossref]

2. Urwin M, Symmons D, Allison T, Brammah T, Busby H, Roxby M (1998) Estimating the burden of musculoskeletal disorders in the community: the comparative prevalence of symptoms at different anatomical sites, and the relation to social deprivation. Annals of the Rheumatic Diseases 57: 649-55. [crossref]

3. Henry J. L (2008) The need for knowledge translation in chronic pain. Pain Res Manag 13: 465-476. [crossref]

4. European Commission (2003) Indicators for monitoring musculoskeletal problems and conditions: musculoskeletal problems and functional limitation. Oslo: University of Oslo.

5. Miranda V. S, Carvalho V. B. F. de, Machado L. A. C, Dias J. M. D. (2012) Prevalence of chronic musculoskeletal disorders in elderly Brazilians: a systematic review of the literature. BMC Musculoskeletal Disorders 13: 82. [crossref]

6. Report by Access Economics Pty Limited for MBF Foundation in collaboration with University of Sydney Pain Management Research Institute. The High Price of Pain: The Economic Impact of Persistent Pain in Australia. Sydney, Australia, 2007.

7. Volkow N. D, McLellan A. T. (2016) Opioid Abuse in Chronic Pain - Misconceptions and Mitigation Strategies. New England Journal of Medicine 374(13): 253-1263. [crossref]

8. Varga Z, Sabzwari S. R. A, Vargova V (2017) Cardiovascular Risk of Nonsteroidal Anti-Inflammatory Drugs: An Under-Recognized Public Health Issue. Cureus 9(4): e1144. [crossref]

9. Cannon P. J. (1986) Prostaglandins in congestive heart failure and the effects of nonsteroidal antiinflammatory drugs. American Journal Medicine 81: 123-132. [crossref]

10. Garcia-Rodriguez L. A, Jick H (1994) Risk of upper gastrointestinal bleeding and perforation associated with individual non-steroidal anti-inflammatory drugs. Lancet 43: 769-772. [crossref]

11. Yamamura Y, Yamamura M (2010) Propedêutica Energética - Inspeção e Interrogatório, 1st edition, Center AO publisher.

12. Zhang R, Lao L, Ren K, Berman B. M (2014) Mechanisms of AcupunctureEletroacupuncture on Persistent Pain. Anesthesiology 120(2): 482-503. [crossref]

13. Yang X. Y, Shi G. X, Li Q. Q, Zhang Z. H, Xu Q, Liu C. Z. (2013) Characterization of Deqi Sensation and Acupuncture Effect. Evid-Based Complement Alternat Med 319734. [crossref]

14. Birch S, Lee M. S, Alraek T, Kim, T. H. (2018) Overview of Treatment Guidelines and Clinical Practical Guidelines That Recommend the Use of Acupuncture: A Bibliometric Analysis. The Journal of Alternative and Complementary Medicine 24(8): 752-769. [crossref]

15. Ministry of Health of Brazil (2006) National Policy of Integrative and Complementary Practices in SUS - PNPIC - SUS: attitude of expanding access. Brasília: Ministry of Health, (Series B. Basic Health Texts).

16. Wirz-Ridolfi. A (2019) The History of Ear Acupuncture and Ear Cartography: Why Precise Mapping of Auricular Points Is Important. Medical Acupuncture 31(3): 145156. [crossref]

17. Aoyama, N, Fujii, O, Yamamoto, T (2017) Efficacy of Parietal Acupoint Therapy: Scalp Acupuncture for Neck/Shoulder Stiffness with Related Mood Disturbance. Medical Acupuncture 29(6): 383-389. [crossref]

18. Wang, J, Pei, J, Khiati, D, Fu, Q, Cui, X, et al. (2017) Acupuncture treatment on the motor area of the scalp for motor dysfunction in patients with ischemic stroke: study protocol for a randomized controlled trial. Trials 18(1): 287. [crossref]

19. Greenspan J. D, Craft R. M, LeResche L, Arendt-Nielsen L, Berkley K. J, et al. Studying sex and gender differences in pain and analgesia: A consensus report. Pain 132: S26-S45. [crossref]

20. El-Shormilisy N, Strong J, Meredith P. J. (2015) Associations among Gender, Coping Patterns and Functioning for Individuals with Chronic Pain: A Systematic Review. Pain Research and Management 20(1): 48-55. [crossref] 
José Udevanier Rebouças da Silva Júnior (2021) Acupuncture Emergency Service in Brazilian Public Health System: Quantitative Analysis of Cases Attended in a Semester

21. Barnett K, Mercer S.W, Norbury M, Watt G, Wyke S, Guthrie B (2012) Epidemiology of multimorbidity and implications for health care, research, and medical education: a cross-sectional study. Lancet 380:37-43. [crossref]

22. Vos T, Allen C, Arora M (2017) Global, regional, and national incidence, prevalence, and years lived with disability for 328 diseases and injuries for 195 countries, 19902016: a systematic analysis for the Global Burden of Disease Study 2016. Lancet 390: 1211-1259.
23. Colvin L. A, Rice A. S. C. (2019) Progress in pain medicine: where are we now? British Journal of Anaesthesia 123(2): e173ee176. [crossref]

24. Chapman C. R, Casey K. L, Dubner R, Foley K. M, Gracely R. H, (1985) Reading, A. E. Pain measurement: an overview. Pain 22(1): 1-31. [crossref]

25. Huskisson E. C. (1974) Measurement of pain. Lancet 2(7889): 1127-31. [crossref]

\section{Citation:}

Rebouças da Silva Júnior JU, Sant’Ana LA, Ziu Lem Gun MC, Bissoto JR, Yamamura Y, et al. (2021) Acupuncture Emergency Service in Brazilian Public Health System: Quantitative Analysis of Cases Attended in a Semester. Prev Med Epid Public Heal Volume 2(4): 1-9. 